PROCEEDINGS OF THE

AMERICAN MATHEMATICAL SOCIETY

Volume 133, Number 11, Pages 3199-3209

S 0002-9939(05)07951-7

Article electronically published on May 9, 2005

\title{
SET-THEORETIC COMPLETE INTERSECTIONS IN CHARACTERISTIC $p$
}

\author{
MARGHERITA BARILE AND GENNADY LYUBEZNIK
}

(Communicated by Bernd Ulrich)

\begin{abstract}
We describe a class of toric varieties which are set-theoretic complete intersections only over fields of one positive characteristic $p$.
\end{abstract}

\section{INTRODUCTION}

The problem of finding a minimal set-theoretic generating set for a toric variety was recently studied in $[2]$ and $[3]$. In [3], in particular, the authors characterized all toric varieties which are set-theoretic complete intersections on binomial equations: a necessary and sufficient condition in characteristic $p>0$ was given in terms of a combinatorial property of the associated semigroup, i.e., the property of being completely $p$-glued, which is based on a notion introduced in 7]. If, however, a toric variety is not completely $p$-glued for some prime $p$, then no general criterion is known so far to decide whether or not it is a set-theoretic complete intersection. In this paper we solve the problem for a class of affine toric varieties whose semigroup is completely $p$-glued only for one prime $p$, by proving that they are settheoretic complete intersections only in characteristic $p$. This provides new examples of the interesting class of varieties for which the minimal number of set-theoretic generators depends on the characteristic. The first known example appeared in [1], where it was shown that the minimum number of set-theoretic defining equations for the variety defined by the vanishing of all $t$-minors of a symmetric matrix, where $t$ is even, is the same in all characteristics except in characteristic 2, where it is strictly smaller.

Received by the editors June 25, 2004.

2000 Mathematics Subject Classification. Primary 14M10, 14M25; Secondary 14F20, 20 M05.

Key words and phrases. Set-theoretic complete intersections, toric varieties, étale cohomology, semigroup gluing.

The first author is grateful to the University of Minnesota for hospitality during the work on this paper.

The second author gratefully acknowledges NSF support.

(C)2005 American Mathematical Society 
Let $K$ be an algebraically closed field. In this paper $V \subset \mathbf{A}_{K}^{n+2}$ denotes a simplicial toric variety parametrized in the following way:

$$
V:\left\{\begin{aligned}
x_{1} & =u_{1}^{p}, \\
x_{2} & =u_{2}^{p}, \\
& \vdots \\
x_{n} & =u_{n}^{p}, \\
y_{1} & =u_{1}^{a_{1}} u_{2}^{a_{2}} \cdots u_{n}^{a_{n}}, \\
y_{2} & =u_{1}^{b_{1}} u_{2}^{b_{2}} \cdots u_{n}^{b_{n}},
\end{aligned}\right.
$$

where $p$ is a prime number.

In Section 1 we prove that $V$ is completely $p$-glued; hence it is a set-theoretic complete intersection in characteristic $p$. Our main result is Theorem 2 stated at the end of Section 2. It implies that there are only two possibilities: either $V$ is a set-theoretic complete intersection in all characteristics, or it is a set-theoretic complete intersection only in characteristic $p$. We give explicit and easily verifiable necessary and sufficient conditions on the exponents $a_{i}$ and $b_{i}$ for each of these two possibilities (conditions (A)-(D) of Section 2). Our proofs (given in Sections 3 and 4) use étale cohomology.

It follows from Theorem 2 that the associated semigroup of $V$ is either completely $q$-glued for all primes $q$, or just for $q=p$, and conditions (A)-(D) of Section 2 provide explicit necessary and sufficient criteria for each of these two possibilities. It is worth pointing out that even though being completely $p$-glued is a combinatorial criterion about a semigroup, which a priori has nothing to do with étale cohomology, the only known (to us) proof of a necessary and sufficient criterion for when the associated semigroup is completely $p$-glued uses étale cohomology. We are unaware of a direct combinatorial proof.

Our results raise a number of open questions. It follows from Theorem 2 that $V$ is a set-theoretic complete intersection in characteristic $p$ if and only if the associated semigroup is completely $p$-glued. This naturally raises the question whether this is true in general, i.e., whether an arbitrary affine toric variety is a set-theoretic complete intersection in characteristic $p$ if and only if the associated semigroup is completely $p$-glued.

Another interesting question is whether there could be varieties which are settheoretic complete intersections in more than one, but not in all positive characteristics, which is certainly not the case for the varieties we study in this paper. It would be especially interesting to know whether there are varieties which are set-theoretic complete intersections in infinitely many but not all characteristics.

\section{1. $V$ IS COMPLETELY $p$-GLUED}

There is a subset $T$ of $\mathbb{N}^{n}$ attached to $V$, namely

$$
\begin{aligned}
T=\{(p, 0,0, \ldots, 0),(0, p, 0, \ldots, 0), & \ldots,(0,0, \ldots, p), \\
& \left.\left(a_{1}, a_{2}, \ldots, a_{n}\right),\left(b_{1}, b_{2}, \ldots, b_{n}\right)\right\} .
\end{aligned}
$$

The polynomials in the defining ideal $I(V)$ of $V$ are the linear combinations of binomials

$$
B_{\alpha_{1}^{-} \alpha_{2}^{-} \cdots \alpha_{n}^{-} \beta_{1}^{-} \beta_{2}^{-}}^{\alpha_{1}^{+} \alpha_{+}^{+} \cdots \alpha_{1}^{+} \beta_{1}^{+} \beta_{1}^{+}} x_{2}^{\alpha_{1}^{+}} x_{2}^{\alpha_{2}^{+}} \cdots x_{n}^{\alpha_{n}^{+}} y_{1}^{\beta_{1}^{+}} y_{2}^{\beta_{2}^{+}}-x_{1}^{\alpha_{1}^{-}} x_{2}^{\alpha_{2}^{-}} \cdots x_{n}^{\alpha_{n}^{-}} y_{1}^{\beta_{1}^{-}} y_{2}^{\beta_{2}^{-}}
$$


with $\alpha_{i}^{+}, \alpha_{i}^{-}, \beta_{i}^{+}, \beta_{i}^{-}$nonnegative integers (not all zero) such that

$$
\begin{aligned}
\alpha_{1}^{+}(p, 0, \ldots, 0) & +\alpha_{2}^{+}(0, p, 0, \ldots, 0)+\cdots+\alpha_{n}^{+}(0,0, \ldots, 0, p) \\
& +\beta_{1}^{+}\left(a_{1}, a_{2}, \ldots, a_{n}\right)+\beta_{2}^{+}\left(b_{1}, b_{2}, \ldots, b_{n}\right) \\
=\alpha_{1}^{-}(p, 0, \ldots, 0) & +\alpha_{2}^{-}(0, p, 0, \ldots, 0)+\cdots+\alpha_{n}^{-}(0,0, \ldots, 0, p) \\
& +\beta_{1}^{-}\left(a_{1}, a_{2}, \ldots, a_{n}\right)+\beta_{2}^{-}\left(b_{1}, b_{2}, \ldots, b_{n}\right) .
\end{aligned}
$$

There is a one-to-one correspondence between the set of binomials in $I(V)$ and the set of semigroup relations $(*)$ between the elements of $T$. Barile, Morales and Thoma proved the following result.

Theorem 1.1 (3], Theorem 5, p. 1899)). An affine or projective toric variety of codimension $r$ over a field $K$ of characteristic $p>0$ is set-theoretically defined by $r$ binomial equations iff it is completely $p$-glued.

The latter notion is due to Rosales [7] and refers to the subgroup of $\mathbb{Z}^{n}$ generated by a set $T$. It is based on the following two definitions, both quoted from [3], pp. $1894-1895$.

Definition 1.2. Let $p$ be a prime number and let $T_{1}$ and $T_{2}$ be nonempty subsets of $T$ such that $T=T_{1} \cup T_{2}$ and $T_{1} \cap T_{2}=\emptyset$. Then $T$ is called a $p$-gluing of $T_{1}$ and $T_{2}$ if there are an integer $k$ and a nonzero element $\mathbf{w} \in \mathbb{Z}^{n}$ such that $\mathbb{Z} T_{1} \cap \mathbb{Z} T_{2}=\mathbb{Z} \mathbf{w}$ and $p^{k} \mathbf{w} \in \mathbb{N} T_{1} \cap \mathbb{N} T_{2}$.

Definition 1.3. An affine semigroup $\mathbb{N} T$ is called completely p-glued if $T$ is the $p$-gluing of $T_{1}$ and $T_{2}$, where each of the semigroups $\mathbb{N} T_{1}, \mathbb{N} T_{2}$ is completely $p$-glued or a free abelian semigroup.

We will say that the variety $V$ is completely $p$-glued if so is the corresponding semigroup $\mathbb{N} T$.

Remark 1.4. Let

$$
T_{1}=\left\{(p, 0,0, \ldots, 0),(0, p, 0, \ldots, 0), \ldots,(0,0, \ldots, p),\left(a_{1}, a_{2}, \ldots, a_{n}\right)\right\}
$$

and consider

$$
T_{11}=\{(p, 0,0, \ldots, 0),(0, p, 0, \ldots, 0), \ldots,(0,0, \ldots, p)\},
$$

which generates a free abelian semigroup, and

$$
T_{12}=\left\{\left(a_{1}, a_{2}, \ldots, a_{n}\right)\right\} .
$$

Then $T_{1}$ is the disjoint union of $T_{11}$ and $T_{12}$ and

$$
\mathbb{Z} T_{11} \cap \mathbb{Z} T_{12}=\mathbb{Z} \mathbf{v}
$$

where

$$
\mathbf{v}= \begin{cases}\left(a_{1}, \ldots, a_{n}\right) & \text { if } p \mid a_{i} \text { for all } i=1, \ldots, n, \\ \left(p a_{1}, \ldots, p a_{n}\right) & \text { otherwise. }\end{cases}
$$

In both cases $\mathbf{v} \in \mathbb{N} T_{11} \cap \mathbb{N} T_{12}$. Hence, for all primes $q, T_{1}$ is the $q$-gluing of $T_{11}$ and $T_{12}$. Moreover, let

$$
T_{2}=\left\{\left(b_{1}, b_{2}, \ldots, b_{n}\right)\right\}
$$

Then

$$
\mathbb{Z} T_{1} \cap \mathbb{Z} T_{2}=\mathbb{Z} \mathbf{w},
$$


where $\mathbf{w}=\lambda\left(b_{1}, \ldots, b_{n}\right)$, and

$$
\lambda=\operatorname{gcd}\left\{k \in \mathbb{N}^{*} \mid\left(k b_{1}, \ldots, k b_{n}\right) \in \mathbb{Z} T_{1}\right\} .
$$

Since $\left(p b_{1}, \ldots, p b_{n}\right) \in \mathbb{N} T_{1}$, it follows that $\lambda=1$ or $\lambda=p$, i.e., $\mathbf{w}=\left(b_{1}, \ldots, b_{n}\right)$ or $\mathbf{w}=\left(p b_{1}, \ldots, p b_{n}\right) \in \mathbb{N} T_{1} \cap \mathbb{N} T_{2}$. Hence $T$ is the $p$-gluing of $T_{1}$ and $T_{2}$ and the variety $V$ given above is completely $p$-glued.

Example 1.5. Consider the following codimension 2 simplicial toric variety of $\mathbf{A}_{K}^{5}$ :

$$
V:\left\{\begin{array}{l}
x_{1}=u_{1}^{2}, \\
x_{2}=u_{2}^{2}, \\
x_{3}=u_{3}^{2}, \\
y_{1}=u_{1}^{2} u_{3}, \\
y_{2}=u_{2}^{2} u_{3} .
\end{array}\right.
$$

If

$$
T_{1}=\{(2,0,0),(0,2,0),(0,0,2),(2,0,1)\}
$$

and

$$
T_{2}=\{(0,2,1)\}
$$

then, with respect to the notation introduced above, $\mathbb{Z} T_{11} \cap \mathbb{Z} T_{12}=\mathbb{Z}(4,0,2)$, since

$$
2(2,0,1)=2(2,0,0)+(0,0,2) .
$$

Moreover $\mathbb{Z} T_{1} \cap \mathbb{Z} T_{2}=\mathbb{Z}(0,2,1)$, since

$$
(0,2,1)=(2,0,0)+(0,2,0)+(0,0,2)-(2,0,1)
$$

and

$$
2(0,2,1)=2(0,2,0)+(0,0,2) \in \mathbb{N} T_{1} \cap \mathbb{N} T_{2},
$$

whereas, evidently, $(0,2,1) \notin \mathbb{N} T_{1} \cap \mathbb{N} T_{2}$. This shows that $V$ is completely 2-glued. It can be easily checked that $V$ is not completely $q$-glued for any other prime $q$. According to Theorem 1 this implies that in characteristic 2 variety $V$ is a settheoretic complete intersection on two binomial equations

$$
F_{1}=y_{1}^{2}-x_{1}^{2} x_{3}, \quad F_{2}=y_{2}^{2}-x_{2}^{2} x_{3},
$$

which are derived from semigroup relations (1.1) and (1.2) respectively, while, in all other characteristics, $V$ cannot be set-theoretically defined by two binomial equations. A complete list of generating binomials for the defining ideal of $V$ is, in every characteristic,

$$
y_{1}^{2}-x_{1}^{2} x_{3}, \quad y_{2}^{2}-x_{2}^{2} x_{3}, \quad x_{1} y_{2}-x_{2} y_{1}, \quad y_{1} y_{2}-x_{1} x_{2} x_{3} .
$$




\section{THE MAIN THEOREM}

If $V$ is not completely $q$-glued for some prime $q$, then, according to Theorem 1.1, in characteristic $q$ it is certainly not a set-theoretic complete intersection on binomial equations, but, in principle, it could still be a set-theoretic complete intersection on some non-binomial equations. The main result of this paper is that for the special kind of toric varieties we are considering this is not the case. In particular, variety $V$ in Example 1.5 is a set-theoretic complete intersection only in characteristic 2 . As a preliminary step, we suppose that $V$ is completely $p$-glued only for one prime $p$ and derive some necessary conditions on the exponents $a_{i}$ and $b_{i}$.

We first remark that the $n$-tuples $\left(a_{1}, a_{2}, \ldots, a_{n}\right)$ and $\left(b_{1}, b_{2}, \ldots, b_{n}\right)$ are such that the support (i.e., the set of indices of nonzero entries) of neither is contained in that of the other: if this condition fails to be true, then $V$ is a set-theoretic complete intersection in every positive characteristic, as is remarked in [3], Example 1. Hence we may assume that

(A) there are indices $i$ and $j$ such that

$$
a_{i}=0, \quad b_{i} \neq 0, \quad \text { and } \quad a_{j} \neq 0, \quad b_{j}=0 .
$$

Moreover

(B) for all $i=1, \ldots, n, \quad p\left|a_{i} \Leftrightarrow p\right| b_{i}$.

In fact, if $p \mid a_{i}$ and $p \nmid b_{i}$ (or vice versa) for some index $i$, then $\left(b_{1}, \ldots, b_{n}\right) \notin$ $\mathbb{Z} T_{1} \cap \mathbb{Z} T_{2}$, so that, by Remark 1.4.

$$
\mathbb{Z} T_{1} \cap \mathbb{Z} T_{2}=\mathbb{Z}\left(p b_{1}, \ldots, p b_{n}\right) .
$$

In this case, since $\left(p b_{1}, \ldots, p b_{n}\right) \in \mathbb{N} T_{1} \cap \mathbb{N} T_{2}$, according to Remark $1.4 \mathbb{N} T$ would be $q$-glued for all primes $q$. The same would be true if for some indices $i, j$ the following linear equation system in $\mathbb{Z} / p \mathbb{Z}$

$$
\left\{\begin{array}{l}
\bar{a}_{i} x+\bar{b}_{i} y=0 \\
\bar{a}_{j} x+\bar{b}_{j} y=0
\end{array}\right.
$$

had only the trivial solution. Hence

(C) for all distinct indices $i, j, \quad p$ divides the minor $\left|\begin{array}{ll}a_{i} & b_{i} \\ a_{j} & b_{j}\end{array}\right|$.

Finally, suppose that all $b_{i}$ 's are divisible by $p$ : then $\left(b_{1}, \ldots, b_{n}\right) \in \mathbb{N} T_{1} \cap \mathbb{N} T_{2}$ generates $\mathbb{Z} T_{1} \cap \mathbb{Z} T_{2}$, and $V$ is completely $q$-glued for all primes $q$. The same argument applies to the $a_{i}$ 's. Hence, taking (B) into account, we conclude that

(D) there is $i$ such that $p \nmid a_{i}$ and $p \nmid b_{i}$.

The main result of this paper is the following:

Theorem 2.1. The following hold for the variety $V$ introduced above.

(a) $V$ is either completely p-glued with respect to only one prime $p$ or completely q-glued with respect to all primes $q$.

(b) $V$ is completely p-glued with respect to only one prime $p$ if and only if conditions (A)-(D) hold.

(c) If conditions (A)-(D) hold, then $V$ is a set-theoretic complete intersection only in characteristic $p$.

It is not hard to show that (c) implies (a) and (b). Indeed, if (c) holds, then, in view of Theorem 1.1 (A)-(D) imply that $V$ is not completely $q$-glued for any 
prime $q \neq p$. Moreover, in this section we have remarked that whenever one of conditions (A)-(D) fails to be true, then $V$ is $q$-glued with respect to all primes $q$. This proves claims (a) and (b). To complete the proof of Theorem 2.1 it remains to prove part (c). This is accomplished in the next two sections. In Section 3 we reduce the proof to the case where $p$ does not divide any $a_{i}$ or $b_{i}$ and in Section 4 we prove part (c) under this additional assumption.

\section{A useful Reduction}

The set-theoretic complete intersection property of the variety $V$ is related to that of certain hyperplane sections of $V$. Fix an index $i, 1 \leq i \leq n$. We consider the following toric variety, whose parametrization is obtained from that of $V$ by omitting the parameter $u_{i}$ :

$$
\bar{V}:\left\{\begin{aligned}
x_{1} & =u_{1}^{p}, \\
x_{2} & =u_{2}^{p}, \\
& \vdots \\
x_{i-1} & =u_{i-1}^{p} \\
x_{i+1} & =u_{i+1}^{p} \\
& \vdots \\
x_{n} & =u_{n}^{p} \\
y_{1} & =u_{1}^{a_{1}} u_{2}^{a_{2}} \cdots u_{i-1}^{a_{i-1}} u_{i+1}^{a_{i+1}} \cdots u_{n}^{a_{n}}, \\
y_{2} & =u_{1}^{b_{1}} u_{2}^{b_{2}} \cdots u_{i-1}^{b_{i-1}} u_{i+1}^{b_{i+1}} \cdots u_{n}^{b_{n}} .
\end{aligned}\right.
$$

$\bar{V}$ is associated with the following subset of $\mathbb{N}^{n-1}$ :

$$
\begin{aligned}
\bar{T}= & \{(p, 0,0, \ldots, 0),(0, p, 0, \ldots, 0), \ldots,(0,0, \ldots, p), \\
& \left.\left(a_{1}, a_{2}, \ldots, a_{i-1}, a_{i+1}, \ldots, a_{n}\right),\left(b_{1}, b_{2}, \ldots, b_{i-1}, b_{i+1}, \ldots, b_{n}\right)\right\} .
\end{aligned}
$$

We introduce some abridged notation. We denote by

$$
\mathbf{e}_{k} \quad \text { the } k \text { th element of the canonical basis of } \mathbb{Z}^{n}
$$

and by

$$
\overline{\mathbf{e}}_{k} \quad \text { the } k \text { th element of the canonical basis of } \mathbb{Z}^{n-1} .
$$

Moreover we set

$$
\begin{aligned}
\mathbf{a}=\left(a_{1}, a_{2}, \ldots, a_{n}\right), & \text { and } & \mathbf{b} & =\left(b_{1}, b_{2}, \ldots, b_{n}\right), \\
\overline{\mathbf{a}}=\left(a_{1}, a_{2}, \ldots, a_{i-1}, a_{i+1}, \ldots, a_{n}\right), & \text { and } \quad & \overline{\mathbf{b}} & =\left(b_{1}, b_{2}, \ldots, b_{i-1}, b_{i+1}, \ldots, b_{n}\right) .
\end{aligned}
$$

Lemma 3.1. Suppose that $p$ divides both the exponents $a_{i}$ and $b_{i}$ in the parametrization of $V$. Let $F=F\left(x_{1}, x_{2}, \ldots, x_{n}, y_{1}, y_{2}\right) \in K\left[x_{1}, x_{2}, \ldots, x_{n}, y_{1}, y_{2}\right]$, and set

$$
\begin{aligned}
\bar{F}=F\left(x_{1}, x_{2}, \ldots, x_{i-1}, 1, x_{i+1}, \ldots, x_{n}, y_{1}, y_{2}\right) & \\
& \quad \in K\left[x_{1}, x_{2}, \ldots, x_{i-1}, x_{i+1}, \ldots x_{n}, y_{1}, y_{2}\right] .
\end{aligned}
$$

Then

$$
F \in I(V) \Longrightarrow \bar{F} \in I(\bar{V})
$$

Conversely, for all $G \in K\left[x_{1}, x_{2}, \ldots, x_{i-1}, x_{i+1}, \ldots, x_{n}, y_{1}, y_{2}\right]$ such that $G \in I(\bar{V})$ there is $F \in K\left[x_{1}, x_{2}, \ldots, x_{n}, y_{1}, y_{2}\right]$ such that $F \in I(V)$ and $\bar{F}=G$. 
Proof. It suffices to prove the claim for binomials. Let $B_{\alpha_{1}^{-} \alpha_{2}^{-} \cdots \alpha_{n}^{-} \beta_{1}^{-} \beta_{2}^{-}}^{\alpha^{+} \alpha^{+} \cdots \alpha^{+} \beta_{+}^{+} \beta^{+}} \in I(V)$. Then the following semigroup relation in $T$ holds:

$(*) \quad \alpha_{1}^{+} p \mathbf{e}_{1}+\alpha_{2}^{+} p \mathbf{e}_{2}+\cdots+\alpha_{n}^{+} p \mathbf{e}_{n}+\beta_{1}^{+} \mathbf{a}+\beta_{2}^{+} \mathbf{b}$

$$
=\alpha_{1}^{-} p \mathbf{e}_{1}+\alpha_{2}^{-} p \mathbf{e}_{2}+\cdots+\alpha_{n}^{-} p \mathbf{e}_{n}+\beta_{1}^{-} \mathbf{a}+\beta_{2}^{-} \mathbf{b} .
$$

It follows that $\overline{B_{\alpha_{1}^{-} \alpha_{2}^{-} \cdots \alpha_{n}^{-} \beta_{1}^{-} \beta_{2}^{-}}^{\alpha_{2}^{+} \alpha_{2}^{+}}} \in I(\bar{V})$, since this binomial corresponds to the following semigroup relation in $\bar{T}$ :

$(* *)$

$$
\begin{aligned}
& \alpha_{1}^{+} p \overline{\mathbf{e}}_{1}+\alpha_{2}^{+} p \overline{\mathbf{e}}_{2}+\cdots+\alpha_{i-1}^{+} p \overline{\mathbf{e}}_{i-1}+\alpha_{i+1}^{+} p \overline{\mathbf{e}}_{i+1}+\cdots+\alpha_{n}^{+} p \overline{\mathbf{e}}_{n}+\beta_{1}^{+} \overline{\mathbf{a}}+\beta_{2}^{+} \overline{\mathbf{b}} \\
& \quad=\alpha_{1}^{-} p \overline{\mathbf{e}}_{1}+\alpha_{2}^{-} p \overline{\mathbf{e}}_{2}+\cdots+\alpha_{i-1}^{-} p \overline{\mathbf{e}}_{i-1}+\alpha_{i+1}^{-} p \overline{\mathbf{e}}_{i+1}+\cdots+\alpha_{n}^{-} p \overline{\mathbf{e}}_{n}+\beta_{1}^{-} \overline{\mathbf{a}}+\beta_{2}^{-} \overline{\mathbf{b}}
\end{aligned}
$$

derived from $(*)$ by skipping the $i$ th component. Conversely, every semigroup relation $(* *)$ in $\bar{T}$ gives rise to the following semigroup relation in $T$ :

$$
\begin{aligned}
& \alpha_{1}^{+} p \mathbf{e}_{1}+\alpha_{2}^{+} p \mathbf{e}_{2}+\cdots+\left(-\beta_{1}^{+} \frac{a_{i}}{p}-\beta_{2}^{+} \frac{b_{i}}{p}\right) p \mathbf{e}_{i}+\cdots+\alpha_{n}^{+} p \mathbf{e}_{n}+\beta_{1}^{+} \mathbf{a}+\beta_{2}^{+} \mathbf{b} \\
& =\alpha_{1}^{-} p \mathbf{e}_{1}+\alpha_{2}^{-} p \mathbf{e}_{2}+\cdots+\left(-\beta_{1}^{-} \frac{a_{i}}{p}-\beta_{2}^{-} \frac{b_{i}}{p}\right) p \mathbf{e}_{i}+\cdots+\alpha_{n}^{-} p \mathbf{e}_{n}+\beta_{1}^{-} \mathbf{a}+\beta_{2}^{-} \mathbf{b} .
\end{aligned}
$$

This proves the second part of the claim.

The following result will be used in the proof of Theorem 2.1.

Lemma 3.2. Suppose that $I(V)=\operatorname{Rad}\left(F_{1}, \ldots, F_{s}\right)$. Then

$$
I(\bar{V})=\operatorname{Rad}\left(\bar{F}_{1}, \ldots, \bar{F}_{s}\right) .
$$

Proof. Inclusion $\supset$ follows from Lemma 3.1, since $I(\bar{V})$ is a reduced ideal. We prove inclusion $\subset$. Let $G \in I(\bar{V})$. By Lemma 3.1 there is $H \in I(V)$ such that $G=\bar{H}$. Then $H^{m} \in\left(F_{1}, \ldots, F_{s}\right)$ for some positive integer $m$, i.e.,

$$
H^{m}=\sum_{i=1}^{s} f_{i} F_{i}, \quad \text { for some } f_{i} \in K\left[x_{1}, x_{2}, \ldots, x_{n}, y_{1}, y_{2}\right] .
$$

Since $\bar{f}_{i} \in K\left[x_{1}, x_{2}, \ldots, x_{i-1}, x_{i+1}, \ldots, x_{n}, y_{1}, y_{2}\right]$, it follows that

$$
G^{m}=\bar{H}^{m}=\overline{H^{m}}=\overline{\sum_{i=1}^{s} f_{i} F_{i}}=\sum_{i=1}^{s} \bar{f}_{i} \bar{F}_{i} \in\left(\bar{F}_{1}, \ldots, \bar{F}_{s}\right),
$$

which completes the proof.

\section{A COHOMOLOGICAL PROOF}

In this section we complete the proof of Theorem 2.1 by proving part (c). We will use étale cohomology $\left(H_{\mathrm{et}}\right)$ and étale cohomology with compact support $\left(H_{\mathrm{c}}\right)$. In the sequel we will assume that char $K \neq p$ and suppose that variety $V$ fulfills conditions (A)-(D). By permuting the indices if necessary we can assume that condition (A) takes the form:

$$
a_{1}=0, \quad b_{2} \neq 0, \quad a_{2} \neq 0, \quad b_{2}=0 .
$$

Condition (B) implies that $p$ divides $a_{2}$ and $b_{1}$. Hence condition (D) implies that there is $i \geq 3$ such that $p \nmid a_{i}$ and $p \nmid b_{i}$. Our aim is to show that $V$ is not set-theoretically defined by two equations. By virtue of Lemma 3.2 it suffices to 
show that this is true for the variety $\bar{V}$ whose parametrization is obtained from that of $V$ by omitting all parameters $u_{i}(3 \leq i \leq n)$ for which $p \mid a_{i}$ (equivalently: $\left.p \mid b_{i}\right)$. Hence conditions (B) and (D) reduce to the following:

(i) $n \geq 3$ and $p$ does not divide $a_{i}$ nor $b_{i}$ for all $i=3,4, \ldots, n$. Condition $(\mathrm{C})$ implies the following:

(ii) for all distinct indices $i, j \geq 3, \quad p$ divides the minor $\left|\begin{array}{cc}a_{i} & b_{i} \\ a_{j} & b_{j}\end{array}\right|$.

Since $p$ divides $a_{2}$ and $b_{1}$, we may adjust the parametrization of $V$ by replacing $u_{1}^{p}, u_{2}^{p}, a_{2} / p$ and $b_{1} / p$ by $u_{1}, u_{2}, a_{2}$ and $b_{1}$ respectively. Then $V$ has the following parametrization:

$$
V:\left\{\begin{aligned}
x_{1} & =u_{1}, \\
x_{2} & =u_{2}, \\
x_{3} & =u_{3}^{p}, \\
& \vdots \\
x_{n} & =u_{n}^{p}, \\
y_{1} & =u_{2}^{a_{2}} u_{3}^{a_{3}} \cdots u_{n}^{a_{n}}, \\
y_{2} & =u_{1}^{b_{1}} u_{3}^{b_{3}} \cdots u_{n}^{b_{n}}
\end{aligned}\right.
$$

and satisfies conditions (i) and (ii). Under these assumptions we prove our claim using the following criterion, cited from [4], Lemma $3^{\prime}$.

Lemma 4.1. Let $W \subset \tilde{W}$ be affine varieties. Let $d=\operatorname{dim} \tilde{W} \backslash W$. If there are $s$ equations $F_{1}, \ldots, F_{s}$ such that $W=\tilde{W} \cap V\left(F_{1}, \ldots, F_{s}\right)$, then

$$
H_{\mathrm{et}}^{d+i}(\tilde{W} \backslash W, \mathbb{Z} / r \mathbb{Z})=0 \quad \text { for all } i \geq s
$$

and for all $r \in \mathbb{Z}$ which are prime to char $K$.

Hence for our purpose it suffices to show that

$$
H_{\mathrm{et}}^{n+4}\left(\mathbf{A}_{K}^{n+2} \backslash V, \mathbb{Z} / p \mathbb{Z}\right) \neq 0 .
$$

For the sake of simplicity, in the sequel we shall omit the coefficient group $\mathbb{Z} / p \mathbb{Z}$. Applying Poincaré Duality (see [6], Cor. 11.2, p. 276) we obtain the equivalent statement:

$$
H_{\mathrm{c}}^{n}\left(\mathbf{A}_{K}^{n+2} \backslash V, \mathbb{Z} / p \mathbb{Z}\right) \neq 0 .
$$

In the long exact sequence

$$
\cdots \longrightarrow H_{\mathrm{c}}^{n-1}\left(\mathbf{A}_{K}^{n+2}\right) \longrightarrow H_{\mathrm{c}}^{n-1}(V) \longrightarrow H_{\mathrm{c}}^{n}\left(\mathbf{A}_{K}^{n+2} \backslash V\right) \longrightarrow H_{\mathrm{c}}^{n}\left(\mathbf{A}_{K}^{n+2}\right) \longrightarrow \cdots
$$

we have that $H_{\mathrm{c}}^{i}\left(\mathbf{A}_{K}^{n+2}\right)=0$ for $i \neq 2 n+4$, so that $H_{\mathrm{c}}^{n}\left(\mathbf{A}_{K}^{n+2} \backslash V\right) \simeq H_{\mathrm{c}}^{n-1}(V)$. Hence we can re-formulate our claim as

$$
H_{\mathrm{c}}^{n-1}(V) \neq 0 .
$$

We prove this by induction on $n$. Note that by (i) necessarily $n \geq 3$. Hence the variety to be considered for the initial step of the induction is

$$
U:\left\{\begin{array}{l}
x_{1}=u_{1}, \\
x_{2}=u_{2}, \\
x_{3}=u_{3}^{p}, \\
y_{1}=u_{2}^{a_{2}} u_{3}^{a_{3}}, \\
y_{2}=u_{1}^{b_{1}} u_{3}^{b_{3}} .
\end{array}\right.
$$

We have to show that

$$
H_{\mathrm{c}}^{2}(U) \neq 0 \text {. }
$$


Now $K[U]=K\left[u_{1}, u_{2}, u_{3}^{p}, u_{2}^{a_{2}} u_{3}^{a_{3}}, u_{1}^{b_{1}} u_{3}^{b_{3}}\right] \subset K\left[u_{1}, u_{2}, u_{3}\right]=K\left[\mathbf{A}_{K}^{3}\right]$. This inclusion corresponds to a map

defined by

$$
\phi: \mathbf{A}_{K}^{3} \rightarrow U
$$

$$
\left(u_{1}, u_{2}, u_{3}\right) \mapsto\left(u_{1}, u_{2}, u_{3}^{p}, u_{2}^{a_{2}} u_{3}^{a_{3}}, u_{1}^{b_{1}} u_{3}^{b_{3}}\right),
$$

which is a finite (hence a proper) morphism. Let $X \subset \mathbf{A}_{K}^{3}$ be the linear subspace defined by $x_{1}=x_{2}=0$. Then $X$ is a one-dimensional affine space. Let $Y=\phi(X)$. We show that $\phi$ induces by restriction a bijection from $\mathbf{A}_{K}^{3} \backslash X$ to $U \backslash Y$. It suffices to show that for all $\left(u_{1}, u_{2}, u_{3}^{p}, u_{2}^{a_{2}} u_{3}^{a_{3}}, u_{1}^{b_{1}} u_{3}^{b_{3}}\right)$ such that $u_{1} \neq 0$ or $u_{2} \neq 0, u_{3}$ is uniquely determined. This is certainly true if $u_{3}=0$. Suppose that $u_{3} \neq 0$. Since $p \nmid a_{3}$ and $p \nmid b_{3}$, there are integers $v, w, s, t$ such that

$$
v p+w a_{3}=1 \quad \text { and } \quad s p+t b_{3}=1 .
$$

If $u_{1} \neq 0$, then

$$
u_{3}=\frac{\left(u_{3}^{p}\right)^{s}\left(u_{1}^{b_{1}} u_{3}^{b_{3}}\right)^{t}}{u_{1}^{b_{1} t}}
$$

if $u_{2} \neq 0$, then

$$
u_{3}=\frac{\left(u_{3}^{p}\right)^{v}\left(u_{2}^{a_{2}} u_{3}^{a_{3}}\right)^{w}}{u_{2}^{a_{2} w}} .
$$

This proves bijectivity. According to [5], Lemma 3.1, bijectivity, together with properness, implies that $\phi$ induces, for all indices $i$, an isomorphism of étale cohomology groups

$$
H_{\mathrm{c}}^{i}\left(\mathbf{A}_{K}^{3} \backslash X\right) \simeq H_{\mathrm{c}}^{i}(U \backslash Y)
$$

From the long exact sequence

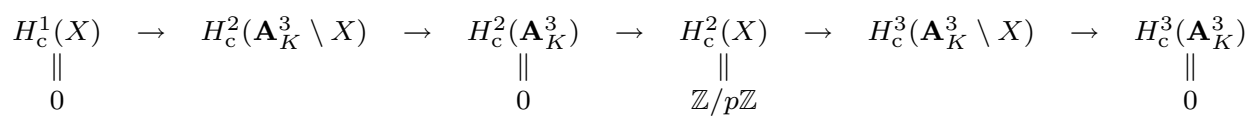

we deduce that $H_{\mathrm{c}}^{2}\left(\mathbf{A}_{K}^{3} \backslash X\right)=0$ and $H_{\mathrm{c}}^{3}\left(\mathbf{A}_{K}^{3} \backslash X\right)=\mathbb{Z} / p \mathbb{Z}$. Moreover, the inclusion

$$
K\left[u_{3}^{p}\right]=K[Y] \subset K[X]=K\left[u_{3}\right]
$$

induces multiplication by $p$ in cohomology with compact support. Since $X$ and $Y$ are both one-dimensional affine spaces, this yields the zero map

$$
\theta: H_{\mathrm{c}}^{2}(Y)=\mathbb{Z} / p \mathbb{Z} \rightarrow \mathbb{Z} / p \mathbb{Z}=H_{\mathrm{c}}^{2}(X),
$$

and, furthermore,

$$
H_{\mathrm{c}}^{3}(Y)=H_{\mathrm{c}}^{3}(X)=0 .
$$

Therefore, in the morphism of complexes induced by the map $\phi$ we have

$$
\begin{aligned}
& \mathbb{Z} / p \mathbb{Z} \quad \mathbb{Z} / p \mathbb{Z} \\
& 0 \rightarrow H_{\mathrm{c}}^{2}(U) \rightarrow H_{\mathrm{c}}^{2}(Y) \stackrel{f}{\rightarrow} \stackrel{\|}{\rightarrow} H_{\mathrm{c}}^{3}(U \backslash Y) \rightarrow H_{\mathrm{c}}^{3}(U) \quad \rightarrow 0 \\
& 0 \rightarrow H_{\mathrm{c}}^{2}\left(\mathbf{A}_{K}^{3}\right) \rightarrow H_{\mathrm{c}}^{2}(X) \rightarrow H_{\mathrm{c}}^{3}\left(\mathbf{A}_{K}^{3} \backslash X\right) \rightarrow H_{\mathrm{c}}^{3}\left(\mathbf{A}_{K}^{3}\right) \rightarrow 0
\end{aligned}
$$

From the commutativity of the central square it follows that map $f$ must be the zero map, which is neither injective nor surjective. Hence

$$
H_{\mathrm{c}}^{2}(U) \neq 0 \quad \text { and } \quad H_{\mathrm{c}}^{3}(U) \neq 0 .
$$


This proves the induction basis. Now assume that $n \geq 4$. Since $p \nmid a_{n}$ and $p \nmid b_{n}$, in particular, we have that $a_{n} \neq 0$ and $b_{n} \neq 0$. Let $W$ be the subvariety of $V$ defined by $x_{n}=0$. Then $W=\mathbf{A}_{K}^{n-1}$, so that from the long exact sequence

$$
\cdots \longrightarrow H_{\mathrm{c}}^{n-2}(W) \longrightarrow H_{\mathrm{c}}^{n-1}(V \backslash W) \longrightarrow H_{\mathrm{c}}^{n-1}(V) \longrightarrow H_{\mathrm{c}}^{n-1}(W) \longrightarrow \cdots
$$

we deduce that $H_{\mathrm{c}}^{n-1}(V) \simeq H_{\mathrm{c}}^{n-1}(V \backslash W)$. Hence our claim (4.1) is equivalent to

$$
H_{\mathrm{c}}^{n-1}(V \backslash W) \neq 0 \text {. }
$$

Now, as a consequence of condition (ii), all maximal minors of the matrix

$$
\left(\begin{array}{cccc}
\bar{a}_{3} & \bar{a}_{4} & \cdots & \bar{a}_{n} \\
\bar{b}_{3} & \bar{b}_{4} & \cdots & \bar{b}_{n}
\end{array}\right)
$$

with entries in $\mathbb{Z} / p \mathbb{Z}$ are 0 ; hence the two rows of the matrix are scalar multiples of each other. Since $n \geq 4$, we can find a positive integer $\lambda_{3}$ such that $p$ divides both $\lambda_{3} a_{3}+a_{4}+\cdots+a_{n-1}+a_{n}$ and $\lambda_{3} b_{3}+b_{4}+\cdots+b_{n-1}+b_{n}$. The coordinate ring of $V \backslash W$ is

$$
\begin{aligned}
K[V \backslash W]= & K\left[u_{n}^{p}, u_{n}^{-p}\right] \otimes_{K} \\
& K\left[\tilde{u}_{1}^{p}, \tilde{u}_{2}^{p}, \tilde{u}_{3}^{p}, \ldots, \tilde{u}_{n-1}^{p}, \tilde{u}_{2}^{a_{2}} \tilde{u}_{3}^{a_{3}} \cdots \tilde{u}_{n-1}^{a_{n-1}}, \tilde{u}_{1}^{b_{1}} u_{3}^{b_{3}} \cdots \tilde{u}_{n-1}^{b_{n-1}}\right],
\end{aligned}
$$

where $\tilde{u}_{3}=u_{3} / u_{n}^{\lambda_{3}}$ and $\tilde{u}_{i}=u_{i} / u_{n}$, for all indices $i \neq 3$. Up to renaming the parameters, thus we have

$$
K[V \backslash W]=K\left[u_{n}^{p}, u_{n}^{-p}\right] \otimes_{K} K\left[u_{1}^{p}, \ldots, u_{n-1}^{p}, u_{2}^{a_{2}} u_{3}^{a_{3}} \cdots u_{n-1}^{a_{n-1}}, u_{1}^{b_{1}} u_{3}^{b_{3}} \cdots u_{n-1}^{b_{n-1}}\right] .
$$

From the Künneth formula for cohomology with compact support (see [6], Theorem 8.5 , p. 258) we deduce that

$$
H_{\mathrm{c}}^{n-1}(V \backslash W) \simeq \bigoplus_{i+j=n-1} H_{\mathrm{c}}^{i}\left(\mathbf{A}_{K}^{1} \backslash\{0\}\right) \otimes H_{\mathrm{c}}^{j}\left(V_{1}\right),
$$

where $V_{1} \subset \mathbf{A}_{K}^{n+1}$ is the affine toric variety parametrized by

$$
V_{1}:\left\{\begin{aligned}
x_{1} & =u_{1}, \\
x_{2} & =u_{2}, \\
x_{3} & =u_{3}^{p}, \\
& \vdots \\
x_{n-1} & =u_{n-1}^{p}, \\
y_{1} & =u_{2}^{a_{2}} u_{3}^{a_{3}} \cdots u_{n-1}^{a_{n-1}}, \\
y_{2} & =u_{1}^{b_{1}} u_{3}^{b_{3}} \cdots u_{n-1}^{b_{n-1}} .
\end{aligned}\right.
$$

Variety $V_{1}$ fulfills (i) and (ii); therefore the induction hypothesis applies to it. Now

$$
H_{\mathrm{c}}^{i}\left(\mathbf{A}_{K}^{1} \backslash\{0\}\right)= \begin{cases}\mathbb{Z} / p \mathbb{Z} & \text { for } i=1,2, \\ 0 & \text { otherwise. }\end{cases}
$$

This, together with (4.3), implies that

$$
H_{\mathrm{c}}^{n-1}(V \backslash W) \simeq H_{\mathrm{c}}^{n-2}\left(V_{1}\right) \oplus H_{\mathrm{c}}^{n-3}\left(V_{1}\right) .
$$

Now, by the induction hypothesis,

$$
H_{\mathrm{c}}^{n-2}\left(V_{1}\right) \neq 0,
$$

because this is claim (4.1) for $V_{1}$. This proves (4.2) and completes the proof of claim (c) and Theorem 2.1. 
Remark 4.2. The induction basis in the proof of Theorem 2.1 strictly depends on the assumption that char $K \neq p$. If char $K=p$, and we take, for some integer $r$ not divisible by $p, \mathbb{Z} / r \mathbb{Z}$ as the coefficient group in cohomology, then map $\theta$ is an isomorphism. Hence the above diagram takes the following form:

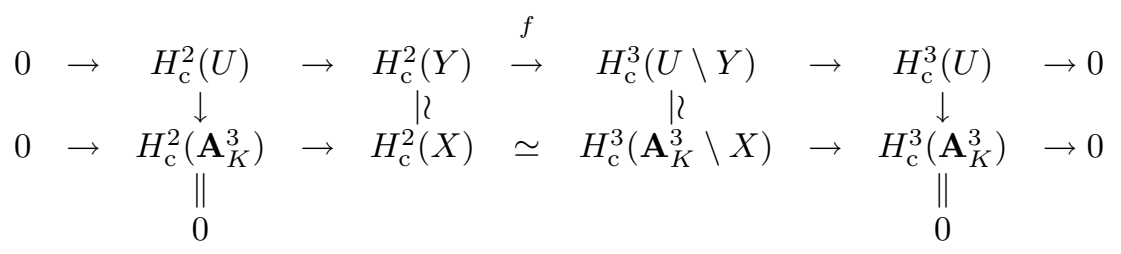

It follows that $f$ is an isomorphism, which implies that $H_{\mathrm{c}}^{2}(U)=H_{\mathrm{c}}^{3}(U)=0$.

\section{REFERENCES}

1. M. Barile, Arithmetical ranks of ideals associated to symmetric and alternating matrices, J. Algebra 176 (1995), 59-82. MR 1345294|(96h:13027)

2. M. Barile, M. Morales, A. Thoma, On Simplicial Toric Varieties Which Are Set-Theoretic Complete Intersections, J. Algebra 226 (2000), 880-892. MF 1752767 (2001i:14066)

3. M. Barile, M. Morales, A. Thoma, Set-Theoretic Complete Intersections on Binomials, Proc. AMS 130 (2002), 1893-1903. MR1896020 (2003f:14058)

4. W. Bruns, R. Schwänzl, The number of equations defining a determinantal variety, Bull. London Math. Soc. 22 (1990), 439-445. MR1082012 (91k:14035)

5. M. Chalupnik, P. Kowalski, Lazard's Theorem for differential algebraic groups and proalgebraic groups, Pac. J. Math. 202 (2002), 305-312. MR.1887766 (2002k:12015)

6. J. S. Milne, Étale cohomology, Princeton University Press, Princeton, 1980. MR0559531 $(81 \mathrm{j}: 14002)$

7. J.C. Rosales, On Presentations of Subsemigroups of $\mathbb{N}^{n}$, Semigroup Forum 55 (1997), 152159. MR $1457760(98 \mathrm{~h}: 20104)$

Dipartimento di Matematica, Università di Bari, Via E. Orabona 4, 70125 Bari, Italy

E-mail address: barile@dm.uniba.it

School of Mathematics, University of Minnesota, 127 Vincent Hall, 206 Church Street S.E., Minneapolis, Minnesota 55455

E-mail address: gennady@math.umn.edu 